\title{
Temperatur in der Praxis lieber rektal messen
}

\author{
Die Temperaturmessungen mit peripher eingesetzten Thermometern sind als Basis für klinische \\ Entscheidungen nicht exakt genug. Die unbeliebte rektale Messung ist deutlich besser.
}

_ Für eine Metaanalyse wurden 75 Studien mit zusammen 8.682 Patienten identifiziert, in denen es um vergleichende periphere Temperaturmessungen im Gehörgang, oral, axillär sowie an der Temporalarterie und zentrale Messungen in der Pulmonalarterie, der Harnblase, im Ösophagus und im Rektum ging. Peripher eingesetzte Thermometer (Ohr, Achsel, Mund, Schläfe) wiesen Messungen außerhalb eines klinisch akzeptablen Bereichs von $\pm 0,5^{\circ} \mathrm{C}$ auf. Das traf besonders für Patienten mit Fieber zu: Hier spannte sich die Abweichung bei Erwachsenen von $-1,44$ bis $+1,46^{\circ} \mathrm{C}$, bei Kindern von $-1,49$ bis $+0,43^{\circ} \mathrm{C}$. Bei der Hypothermie war die Abweichung mit $-2,07$ bis $+1,90^{\circ} \mathrm{C}$ bei den Erwachsenen noch größer. Die Sensitivität zur Feststellung von Fieber lag für periphere Thermometer nur bei $64 \%$, bei allerdings hoher Spezifität von $96 \%$.

Die beste Korrelation mit dem Goldstandard, der Messung über einen Pulmonaliskatheter, weisen die Messung im Rektum und in der Blase (über einen lie- genden Katheter) auf. Als zweitbeste Messpunkte empfehlen die Autoren bei Kindern das Ohr und bei Erwachsenen unter der Zunge.

- Niven DJ et al. Accuracy of peripheral thermometers for estimating temperature: a systematic review and metaanalysis. Ann Int Med. 2015;163:768-77

\section{KOMMENTAR}

Man wundert sich schon, dass eine derart basales klinisches Problem erst jetzt systematisch untersucht wurde.

Prof. Dr. med. H. S. FüeßI

\section{EKG-Ableitung weist den Weg zur Diagnose eines Wellens-Syndroms}
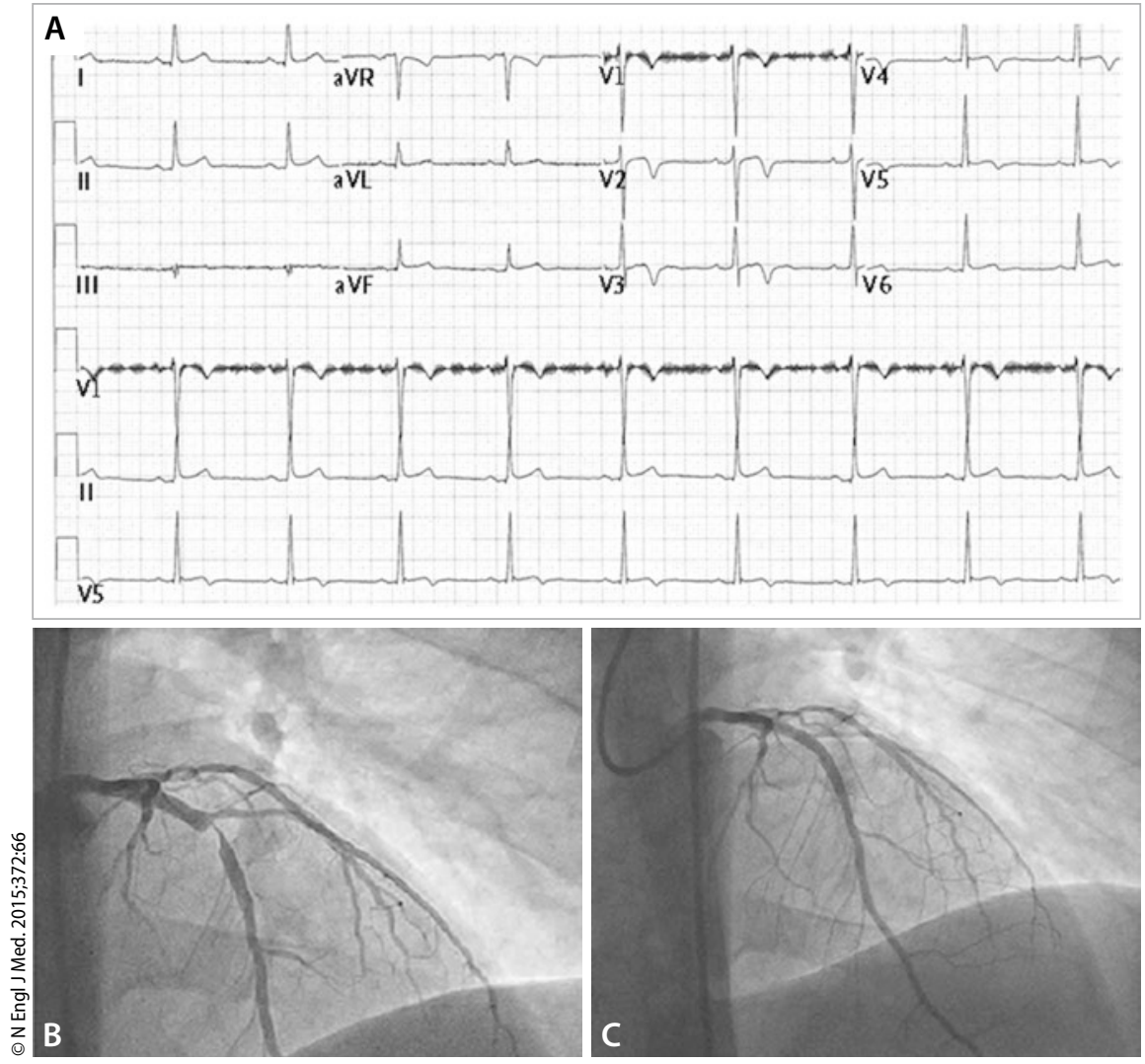

EKG mit anterolateraler T-Inversion und biphasischen T-Wellen im Lateralbereich (A), Stenose der LAD in der Angiografie (B), Zustand nach Versorgung mit Stent (C).
Ein 31-jähriger Raucher mit Diabetes mellitus stellte sich mit seit vier Tagen bestehenden, belastungsabhängigen Brustschmerzen in der Nothilfe vor. Das EKG bei Aufnahme zeigte eine anterolaterale T-Inversion mit biphasischen TWellen im Lateralbereich (Abb. A). Aufgrund dieser Konstellation stellte man die Verdachtsdiagnose eines Wellens-Syndroms. Dieses Syndrom ist assoziiert mit einer hochgradigen Stenose der LAD. (Der Artefakt in Ableitung V1 hat damit nichts zu tun). Die Troponin-I-Konzentration war bei Aufnahme mit $0,07 \mathrm{ng} / \mathrm{ml}$ leicht erhöht und stieg sechs Stunden später auf 0,58 $\mathrm{ng} / \mathrm{ml}$. Die Koronarangiografie nach 12 Stunden zeigte eine 95\%ige Stenose der LAD im mittleren Bereich (Abb. B), die erfolgreich mit einem beschichteten Stent versorgt wurde (Abb. C). Der Diabetes des Patienten war schlecht kontrolliert, sein $\mathrm{HbA}_{1 c}$-Wert lag bei $11,9 \%$.

Nach der Intervention erholte sich der Patient rasch, die linksventrikuläre Ejektionsfraktion war voll erhalten. Er wurde nach einem Rauchstopp-Training und einer intensiven Diabetesschulung in die kardiologische Rehabilitation entlassen. Da kann man nur sagen: Noch einmal Glück gehabt.

Prof. Dr. med. H. S. FüeßI

- Kannan L, Figueredo VM (kannanla@einstein.edu). Wellens' Syndrome. N Engl J Med. 2015;372:66 